\title{
Synthesis, Thermal Stability, and Light-Emitting Properties of Hyperbranched Poly(phenylenegermolene)s
}

\author{
Charles C. W. Law, ${ }^{1}$ Junwu Chen, ${ }^{1}$ Jacky W. Y. Lam, ${ }^{1}$ Han Peng, ${ }^{1}$ and \\ Ben Zhong Tang ${ }^{1,2}$
}

Received August 3, 2003

\begin{abstract}
A germole-containing diacetylene, 1,1-diethynyl-2,3,4,5-tetraphenylgermole (2), was synthesized. Homopolymerization of $\mathbf{2}$ and its copolycyclotrimerization with 1-octyne were effected by $\mathrm{TaCl}_{5}-\mathrm{Ph}_{4} \mathrm{Sn}$ in toluene, producing completely soluble polymers (1) in moderate yields. The molecular structure of $\mathbf{1}$ was characterized by spectroscopic methods. The thermal stability of 1 was evaluated by thermogravimetric analysis, which detected virtually no weight loss when the polymers were heated to $\sim 350^{\circ} \mathrm{C}$. These hyperbranched polymers were electronically conjugated as suggested by their strong absorption in the visible spectral region $\left(\lambda_{\max } \sim 540 \mathrm{~nm}\right)$. Photoluminescence of the polymers was dramatically enhanced by cooling their solutions to low temperatures. Restricted intramolecular rotations of the phenyl rings upon the axes of the single bonds linked to the germole cores at the cryogenic temperatures might be responsible for this unique phenomenon of cooling-enhanced emission.
\end{abstract}

KEY WORDS: Germole; hyperbranched polyphenylenes; alkyne polycyclotrimerization; luminescence; cooling-enhanced light emission.

\section{INTRODUCTION}

Design and synthesis of conjugated polymers have attracted much attention in recent decades owing to potential applications of the polymers in electronics and optics [1,2]. Our group has embarked on a research program with the aim of developing polyacetylenes with chromophoric

\footnotetext{
${ }^{1}$ Department of Chemistry, Center for Display Research, Institute of Nano Science and Technology, and Open Laboratory of Chirotechnology, Hong Kong University of Science \& Technology, Clear Water Bay, Kowloon, Hong Kong, China.

${ }^{2}$ To whom correspondence should be addressed. E-mail: tangbenz@ust.hk
} 
pendants [3-7]. During our search for efficient light-emitting materials, we discovered a group of highly luminescent organometallic molecules called siloles. We found that the silole molecules and polymers exhibited interesting photoluminescence (PL) properties such as aggregation-induced emission (AIE) [8-14]. They are practically non-emissive when molecularly dissolved, but their nanoaggregates in poor solvents (e.g., a $90 \%$ methanol/ chloroform mixture) and thin solid films are highly luminescent; that is, their emissions are induced by aggregate formation. The PL can also be significantly boosted by cooling their solutions, which may be a result of restricted intramolecular rotations of the phenyl peripheries around the single bonds linked to the silole core at the low temperatures.

Similar to silicon, germanium is also a metalloid element bearing both metallic and nonmetallic characteristics. Few papers are, however, available in the literature reporting the synthesis and properties of germoles. In this paper, we have extended our synthetic efforts to include the preparation of hyperbranched polyphenylenes that contain germole moieties. We utilized the diyne polycyclotrimerization procedure that we recently developed [15-18] and succeeded in the synthesis of hyperbranched poly(phenylenegermolene)s (1) (Scheme 1). All the polymers are completely soluble in common organic solvents such as tetrahydrofuran (THF), chloroform, and toluene, thanks to their highly branched molecular structures. The PL of the polymers are weak at room temperature, but can be greatly enhanced by cooling their solutions to low temperatures.

\section{EXPERIMENTAL}

\section{Reagents and Solvents}

THF and toluene were pre-dried over $4 \AA$ molecular sieves and distilled from sodium benzophenone ketyl immediately prior to use. Diphenylacetylene (tolan; 4), germanium(IV) chloride, lithium wire, 1-octyne, ethynylmagnesium bromide $(0.5 \mathrm{M}$ in THF), tantalum(V) chloride, and tetraphenyltin were purchased from Aldrich and were used as received without further purification.

\section{Characterization Techniques}

Average molecular weights $\left(M_{\mathrm{w}}\right.$ and $\left.M_{\mathrm{n}}\right)$ were estimated by a Waters 510 gel permeation chromatograph (GPC). A set of 12 monodisperse polystyrene standards covering molecular weight range of $10^{2}-10^{7}$ was used for the molecular weight calibration. IR spectra were recorded on a Perkin 

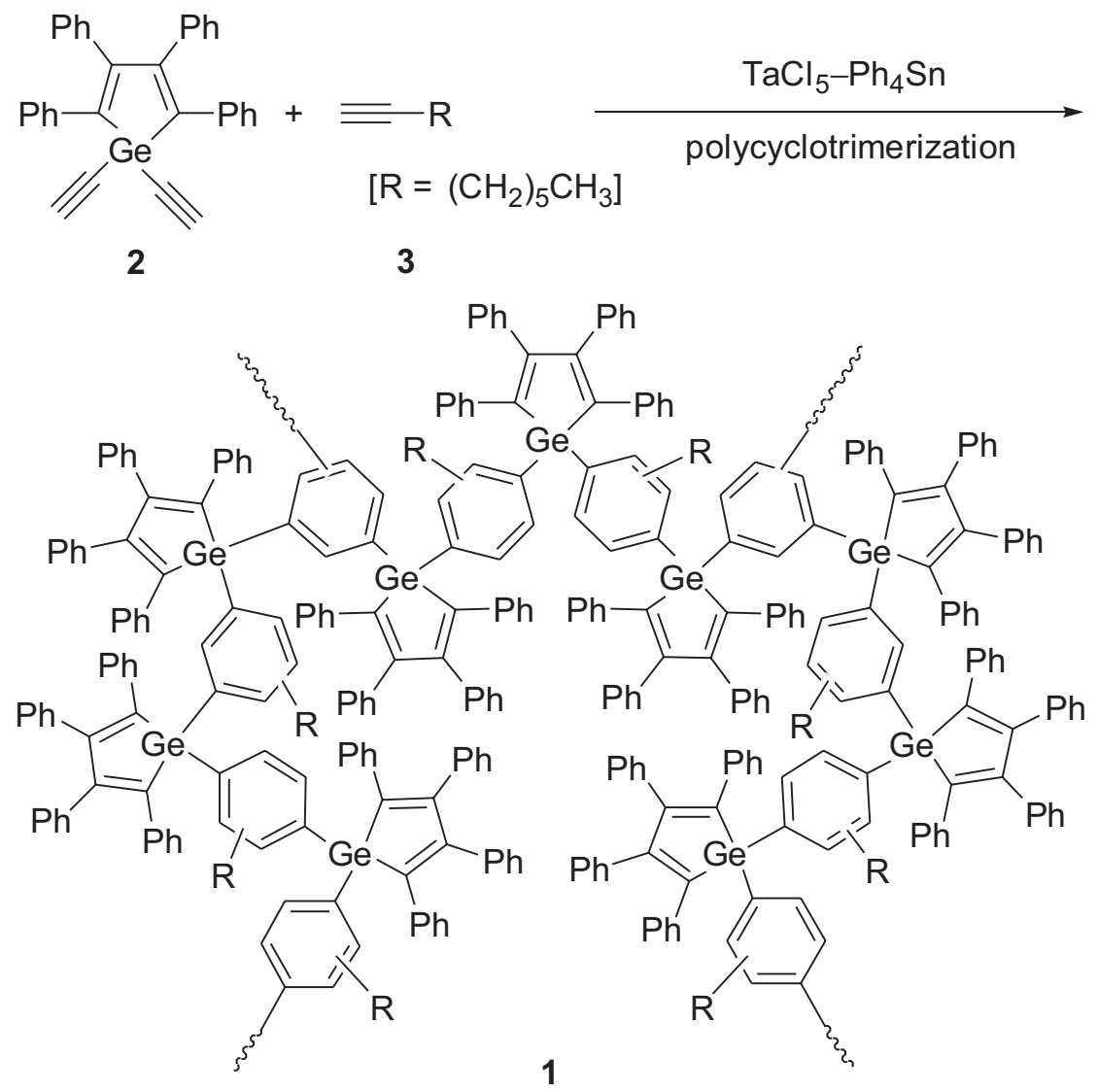

Scheme 1.

Elmer 106 PC FTIR spectrometer. ${ }^{1} \mathrm{H}-\mathrm{NMR}$ spectra were obtained on a Bruker ARX 300 spectrometer using tetramethylsilane (TMS) as an internal reference. Thermal stability was evaluated with a Perkin-Elmer thermogravimetric analyzer TGA 7. The experiments were carried out under nitrogen at a heating rate of $20^{\circ} \mathrm{C} / \mathrm{min}$. UV absorption spectra were recorded with a Milton Roy Spectronic 3000 Array spectrophotometer. PL spectra of the polymer solutions and the nanoaggregate suspensions were recorded with a SLM 8000C spectrometer. The nanoaggregate suspensions were freshly prepared by adding poor solvents into the solutions of the polymers in good solvents with vigorous shaking. The concentrations of all the nanoaggregates were adjusted to $12 \mu \mathrm{g} / \mathrm{mL}$. 


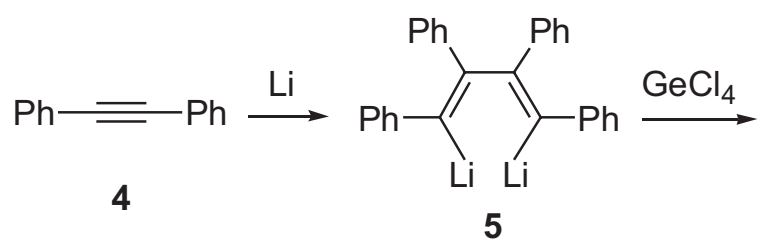

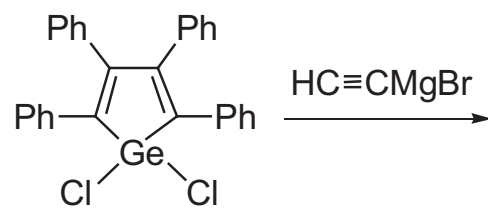

6

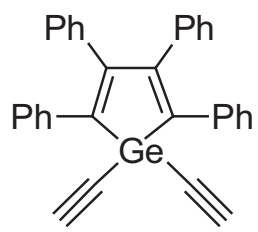

2

Scheme 2.

\section{Synthesis}

The germolyldiyne monomer (2) was prepared by the reaction of 1,1dichloro-2,3,4,5-tetraphenylgermole (6) with ethynylmagnesium bromide (Scheme 2). The detailed synthetic procedures are given below.

1,1-Dichloro-2,3,4,5-Tetraphenylgermole (6). Under pure, dry nitrogen, fresh lithium shavings $(150 \mathrm{mg}, 21.6 \mathrm{mmol})$ were added to a solution of $4(5 \mathrm{~g}, 28.0 \mathrm{mmol})$ in THF $(25 \mathrm{~mL})$. The mixture was stirred for $12 \mathrm{~h}$ at room temperature and then added dropwise to a solution of germanium(IV) chloride $(0.88 \mathrm{~mL}, 7.56 \mathrm{mmol})$ in THF $(120 \mathrm{~mL})$. The solution was stirred for $2 \mathrm{~h}$ at room temperature and then refluxed for $5 \mathrm{~h}$ at elevated temperatures, which gave the desired intermediate 6.

1,1-Diethynyl-2,3,4,5-Tetraphenylgermole (2). Into the above THF solution of 6 at room temperature, a THF solution of ethynylmagnesium bromide $(36.0 \mathrm{~mL}, 0.5 \mathrm{M}, 18.1 \mathrm{mmol})$ was added dropwise. After stirring for $2 \mathrm{~h}$, the mixture was filtered and the crude product was purified on a silica-gel column using hexane/chloroform (3:1 by volume) as the eluent. Recrystallization from toluene/heptane mixture gave a pale yellowishgreen solid; Yield, $1.16 \mathrm{~g}$ ( $32 \%$ based on germanium(IV) chloride). IR $(\mathrm{KBr}), v\left(\mathrm{~cm}^{-1}\right): 3257$ ( $\equiv \mathrm{C}-\mathrm{H}$ stretch), 2039 (C $\equiv \mathrm{C}$ stretch). ${ }^{1} \mathrm{H}-\mathrm{NMR}$ (300 $\mathrm{MHz}, \mathrm{CDCl}_{3}$ ), $\delta$ (ref. TMS, ppm), (multiplicity, integration): 6.81-7.15 (m, 20H), $2.58(\mathrm{~s}, 2 \mathrm{H}) .{ }^{13} \mathrm{C}-\mathrm{NMR}\left(75 \mathrm{MHz}, \mathrm{CDCl}_{3}\right), \delta$ (ref. TMS, ppm): 137.3, 131.4, 129.6, 129.5, 128.0, 127.8, 126.8, 126.7, 126.6, $125.2,95.7$ ( $\equiv \mathrm{C}), 81.2(\mathrm{HC} \equiv)$. 


\section{Polymerization}

All the polymerization reactions and manipulations were carried out under nitrogen using a standard Schlenk technique in a vacuum line system or an inert-atmosphere glovebox (Vacuum Atmospheres), except for the purification of the polymers, which were conducted in open air. A typical procedure for the copolymerization of 2 with 1-octyne (3) is given below.

Into a thoroughly baked and moisture excluded Schlenk tube were placed $\mathrm{TaCl}_{5}(18.0 \mathrm{mg}, 0.05 \mathrm{mmol})$ and $\mathrm{Ph}_{4} \mathrm{Sn}(21.3 \mathrm{mg}, 0.05 \mathrm{mmol})$. Loading of the reactants was carried out in a glovebox. The catalyst mixture was dissolved in toluene $(1.0 \mathrm{~mL})$ and aged at room temperature for $15 \mathrm{~min}$. A solution of $2(85.0 \mathrm{mg}, 0.18 \mathrm{mmol})$ and $3(13.3 \mu \mathrm{L}, 0.09 \mathrm{mmol})$ in toluene $(1.5 \mathrm{~mL})$ was then added dropwise into the catalyst solution. After stirring at room temperature for $24 \mathrm{~h}$, the reaction was quenched by the addition of a small amount of methanol. The polymer solution was dropped into methanol $(250 \mathrm{~mL})$ via a cotton filter with stirring. A deep purple powder of $1 \mathrm{~b}$ was isolated; Yield, $41 \% ; M_{\mathrm{w}}, 4450 ; M_{\mathrm{w}} / M_{\mathrm{n}}, 8.45$. ${ }^{1} \mathrm{H}-\mathrm{NMR}$ (300 MHz, CDCl3), $\delta$ (ref. TMS, ppm): 6.84, 1.25, 0.86.

\section{RESULTS AND DISCUSSION}

\section{Monomer and Polymer Syntheses}

Germolyldiyne was synthesized by attaching two ethynyl groups to the 1,1-position of germacyclopentadiene ring (Scheme 2). Lithiation of tolan 4 gave 1,4-dilithiotetraphenylbutadiene 5 . Cyclization of 5 with germanium(IV) chloride yielded 1,1-dichloro-2,3,4,5-tetraphenylgermole 6 [19]. The nucleophilic substitution of $\mathbf{6}$ with a Grignard reagent, ethynylmagnesium bromide, led to the formation of the desired monomer 2 . The molecular structure of $\mathbf{2}$ was characterized by standard spectroscopic methods, from which satisfactory analysis data were obtained.

Polycyclotrimerization of 2 was effected with $\mathrm{TaCl}_{5}-\mathrm{Ph}_{4} \mathrm{Sn}$ in toluene at room temperature, which gave a hyperbranched polyphenylene (1a) in $47 \%$ yield. Despite the all-aromatic nature of the polymer 1a, it is completely soluble in common solvents such as THF, chloroform, toluene, and dichloromethane, thanks to its highly branched molecular structure. The GPC analysis using linear polystyrenes as calibration standards gave a weight-average molecular weight of 5160 . This value is probably considerably underestimated because of the hyperbranched nature of the polymer coupled with its rigid molecular structure. In our previous studies on hyperbranched polymers, we found that underestimation could be as large as 7-fold. Therefore, the actual molecular weight of 1a could be much higher than the relative value estimated from the GPC analysis. 
Table I. Polymerization of Germolyldiyne $2^{a}$

\begin{tabular}{cccccc}
\hline No. & {$[2](\mathrm{M})$} & {$[3](\mathrm{M})$} & yield $(\%)$ & $M_{\mathrm{w}}{ }^{b}$ & $M_{\mathrm{w}} / M_{\mathrm{n}}{ }^{b}$ \\
\hline 1a & 0.1 & 0 & 47 & 5160 & 1.78 \\
1b & 0.1 & 0.05 & 41 & 4450 & 8.45 \\
\hline
\end{tabular}

${ }^{a}$ Carried out under nitrogen at room temperature in toluene using $\mathrm{TaCl}_{5}-\mathrm{Ph}_{4} \mathrm{Sn}$ as catalyst; $\left[\mathrm{TaCl}_{5}\right]=\left[\mathrm{Ph}_{4} \mathrm{Sn}\right]=10 \mathrm{mM}$.

${ }^{b}$ Estimated by GPC in THF on the basis of a polystyrene calibration.

Copolymerization of diyne $\mathbf{2}$ with monoyne 3 in a molar feed ratio, $[3] /[2]=0.5$, produced a copolymer (1b) with a similar molecular weight (4450). Like its homopolymer counterpart, $\mathbf{1 b}$ is also completely soluble and readily processible, forming thin films upon spin-coating its solutions onto solid substrates.

\section{Characterization by Spectroscopic Methods}

All the polymers gave satisfactory spectroscopic data corresponding to their expected molecular structures. An example of the IR spectra of 1a and $\mathbf{1 b}$ is given in Fig. 1. The spectrum of monomer 2 is given in the same figure for the purpose of comparison. As can be seen in the spectrum of 2, the $\equiv \mathrm{C}-\mathrm{H}$ and $\mathrm{C} \equiv \mathrm{C}$ stretching vibrations occur at $3257 \mathrm{~cm}^{-1}$ and

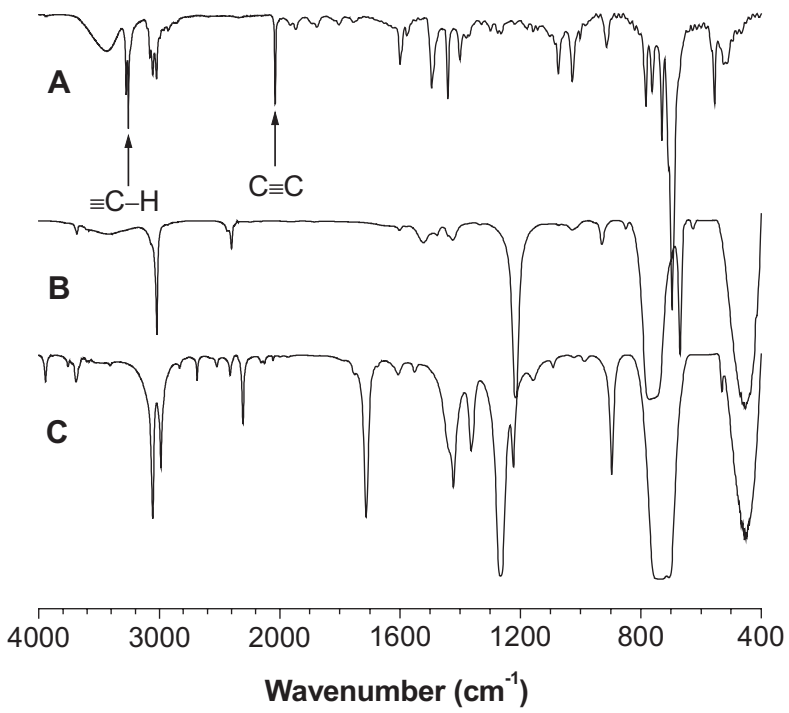

Fig. 1. IR spectra of (A) monomer 2, and (B) homopolymer 1a, and (C) copolymer $\mathbf{1 b}$. 
$2039 \mathrm{~cm}^{-1}$, respectively. These absorption bands, however, are not observed in the spectra of $\mathbf{1 a}$ and $\mathbf{1 b}$, and indicate that the hyperbranched polymers contain no triple bonds. The triple bonds may have been exhausted by the cyclotrimerization reactions, or the alkyne building blocks have been consumed in the construction of the aromatic cores and peripheries.

Figure 2 shows the ${ }^{1} \mathrm{H}-\mathrm{NMR}$ spectra of copolymer $\mathbf{1 b}$, diyne $\mathbf{2}$, and monoyne 3 in chloroform- $d$. The acetylenic protons of 3 (a; Fig. 2A) and 2 (e; Fig. 2B) resonate at $\delta 1.92$ and 2.58, respectively. These peaks are, however, absent in the spectrum of $\mathbf{1 b}$ (Fig. 2C). The polymerization reaction converts the acetylene triple bonds to the aromatic rings, which give a strong peak for the aryl protons in the downfield region. The aryl absorption peak is, however, structureless and prevents an estimation of the isomeric ratio of the 1,2,4- and 1,3,5-conformers in the polymer. As a result of polycyclotrimerization, the propargyl protons $(b)$ of $\mathbf{3}$ are transformed to the benzyl protons $\left(b^{\prime}\right)$ of $\mathbf{1 b}$. The two partially overlapping peaks in the spectral region of $\delta 0.7-1.8$ are due to the resonance of the aliphatic protons of the pentyl group contributed by the monoyne 3 .

\section{Thermal Stability and Electronic Absorption}

All the germole-containing hyperbranched polymers are thermally stable; that is, no weight losses are detected by TGA analyses when the polymers are heated to $\sim 300^{\circ} \mathrm{C}$ (Fig. 3). About $30-40 \%$ of their weight remains when the polymers are heated to $\sim 800^{\circ} \mathrm{C}$; i.e., the polymers graphitize upon pyrolysis.

Figure 4 shows the UV-visible absorption spectra of monomer 2 and the polymers 1a and 1b. Monomer $\mathbf{2}$ exhibits a strong absorption peak at $\sim 370 \mathrm{~nm}$ due to $\pi-\pi^{*}$ transitions of the germacyclopentadiene ring [10]. Little absorption is observed in the spectral region beyond $440 \mathrm{~nm}$. The hyperbranched polymers, however, absorb in the visible region. This suggests that the polymers possess extended conjugation, possibly due to the synergistic interplay of the $\sigma-\pi$ conjugation of the germole rings and the electronic communication of the aromatic rings via the germanium bridges [20-22]. The homopolymer 1a is more conjugated, as judged from its stronger absorption in the visible region $\left(\lambda_{\max } \sim 540 \mathrm{~nm}\right)$. Copolymer $1 \mathbf{b}$ is less conjugated, probably owing to the incorporation of the non-conjugating 1-octyne moieties into the hyperbranched polymer structure.

\section{Light Emission}

As discussed above, we have previously found that the emission spectrum of silole molecules and their polymers can be enhanced by 
(A)
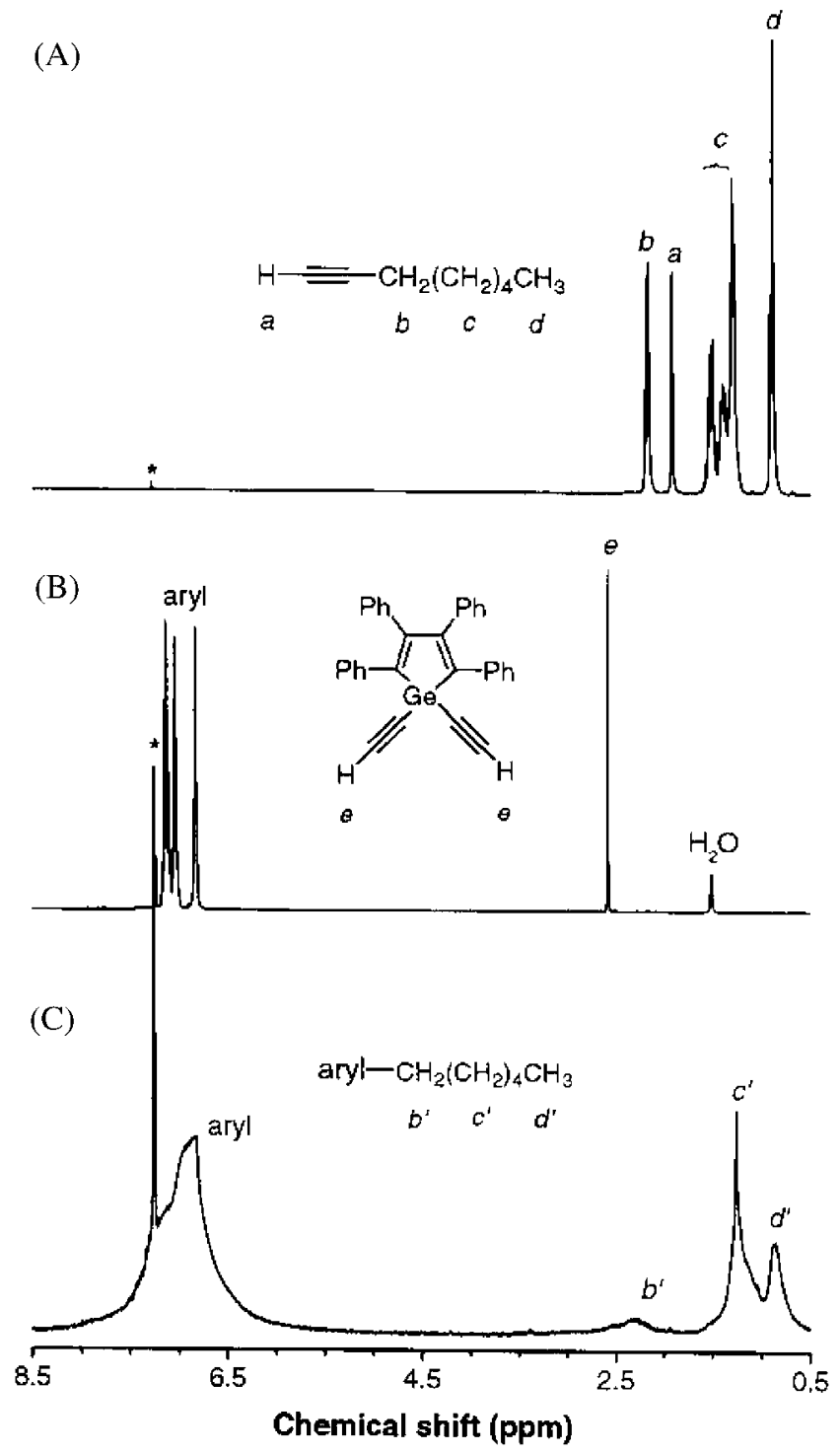

Fig. 2. ${ }^{1} \mathrm{H}-\mathrm{NMR}$ spectra of (A) 1-octyne 3, (B) germolyldiyne 2 , and (C) their polymer $\mathbf{1 b}$ in chloroform- $d$. The solvent peaks are marked with asterisks $(*)$. 


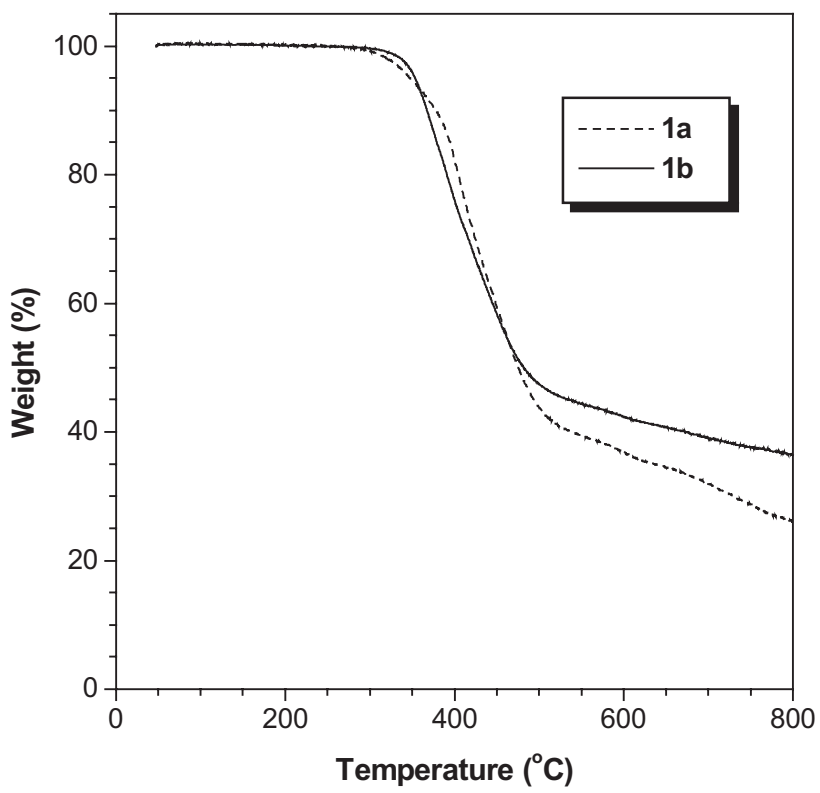

Fig. 3. TGA thermograms of homopolymer 1a and copolymer $\mathbf{1 b}$ recorded under nitrogen at a heating rate of $20^{\circ} \mathrm{C}$.

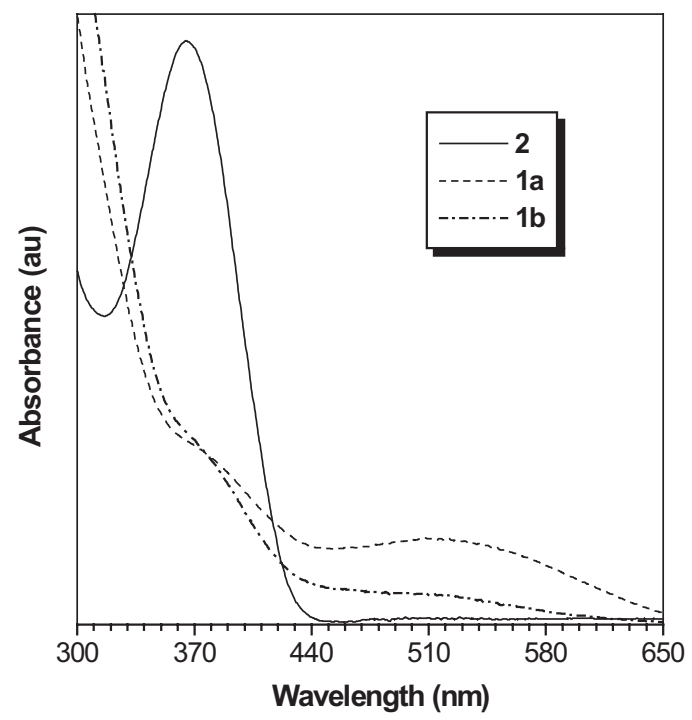

Fig. 4. UV spectra of chloroform solutions of monomer $\mathbf{2}$ and its homopolymer $\mathbf{1 a}$ and copolymer $\mathbf{1 b}$. 
aggregation and cooling [11-14]. A reasonable question is "since germanium is in the same group as silicon, would 2 and its polymers exhibit similar spectral behavior?" When a chloroform solution of 2 is photoexcited at $410 \mathrm{~nm}$, a blue light at $474 \mathrm{~nm}$ is emitted. Using rhodamine B as a standard, the quantum yield $\left(\Phi_{\mathrm{F}}\right)$ of the chloroform solution is calculated to be $1 \%$, which is much higher than that of silole with the same structure $\left(\Phi_{\mathrm{F}} \leqslant 0.1 \%\right)$. When methanol, a non-solvent of 2 , is added, the emission becomes stronger (Fig. 5); that is, germole, like silole, is also AIE-active. The emission spectrum of both the silicon- and germanium-containing polymers is in a similar wavelength region. Whereas $\Phi_{\mathrm{F}}$ of $1 \mathrm{a}$ is $1 \%, \Phi_{\mathrm{F}}$ of 1b is 5-fold greater. Although the polymers are luminescent, they are, in general terms, still weak emitters. Surprisingly, the emission from a methanol/ THF mixture (9:1 by volume) of 1a is much weaker than that of pure chloroform solution. In our previous study, we found that when the silole pendant groups were directly attached to the rigid linear polyacetylene backbone, the resultant polymer was AIE-inactive [13]. In this study, the germole rings are placed into the rigid hyperbranched polyphenylene sphere and the resultant polymers are again AIE-inactive. The stiff polyacetylene backbone and the hard polyphenylene sphere may have piled up randomly

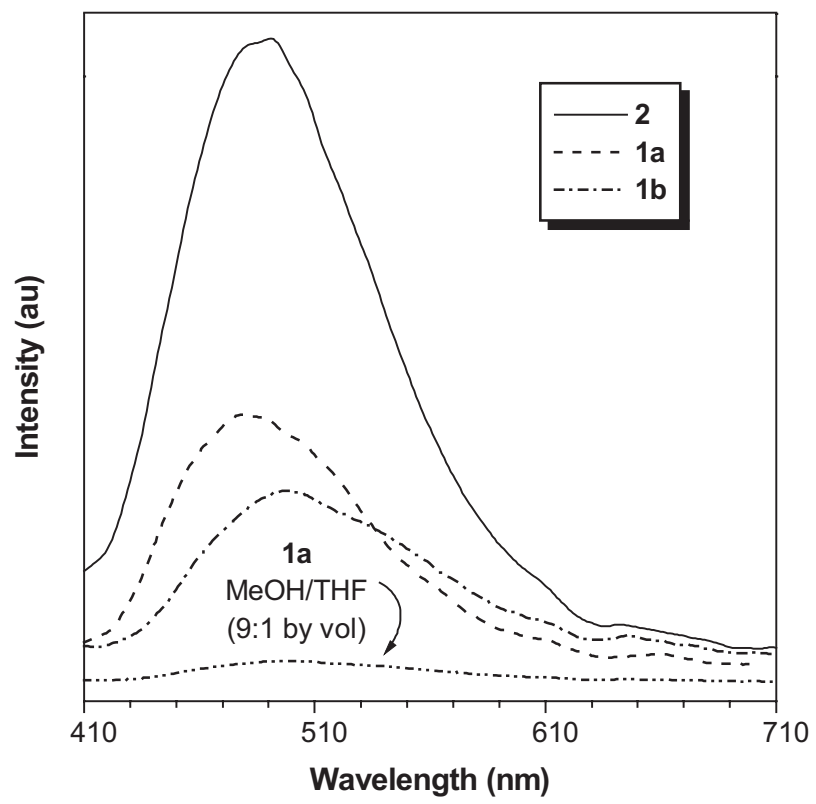

Fig. 5. Photoluminescence spectra of $\mathbf{2}, \mathbf{1 a}$, and $\mathbf{1 b}$ in chloroform and $\mathbf{1 a}$ in a methanol/THF mixture (9:1 by volume) at room temperature; $[1]=[2]=12 \mu \mathrm{g} / \mathrm{mL}, \lambda_{\mathrm{ex}}=410 \mathrm{~nm}$. 


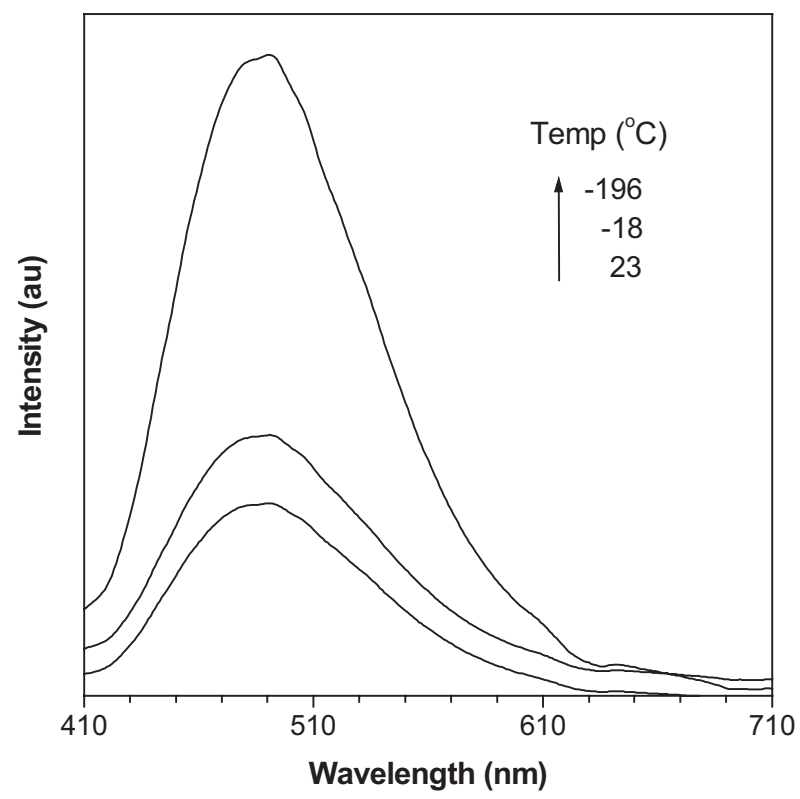

Fig. 6. Photoluminescence spectra of $\mathbf{1 a}$ in THF at different temperatures; [1a] $=12 \mu \mathrm{g} / \mathrm{mL}$, $\lambda_{\mathrm{ex}}=410 \mathrm{~nm}$.

when they aggregate in the poor solvents. Such chaotic stacks of the rigid polymers may generate large free volumes in the aggregates [23-26], in which the phenyl rings may still be able to rotate around the axes of the single bonds that are linked to the germacyclopentadiene core. Such intramolecular rotations may have non-radiatively deactivated the excited species $[27,28]$ and, thus, quench the emission from the germole pendant groups.

It was then checked whether cooling the polymer could enhance emission. When a dilute THF solution of $1 \mathrm{a}$ is cooled from room temperature to $-18^{\circ} \mathrm{C}$, the PL intensity is increased (Fig. 6). It is suspected that intramolecular rotations of the phenyl rings round the axes of the germacyclopentadiene core may be impeded at the low temperature, resulting in stronger emission. The spectral profile hardly changes when the temperature is lowered further to $-196^{\circ} \mathrm{C}$, which suggests that the PL is still associated with the radiative decay of the singlet excitons but not the triplet species.

\section{CONCLUSIONS}

In this work, we succeeded in incorporating the germole ring, an organometallic chromophore with novel luminescence properties, into the 
hyperbranched polyphenylene structure through facile polycyclotrimerization of alkynes catalyzed by $\mathrm{TaCl}_{5}-\mathrm{Ph}_{4} \mathrm{Sn}$ under mild reaction conditions. The germolyl rings are linked together by the phenyl rings in three-dimensional space. This unique molecular structure endows 1 with novel properties; i.e., the polymers are thermally stable $\left(>350^{\circ} \mathrm{C}\right)$, electronically conjugated $\left(\lambda_{\max } \sim 540 \mathrm{~nm}\right)$, and highly emissive upon excitation at low temperatures.

\section{ACKNOWLEDGMENTS}

The work described in this paper was partially supported by the Hong Kong Research Grants Council (HKUST 6121/01P, 6085/02P, and 6049/03P) and the University Grants Committee of Hong Kong through an Area of Excellence Scheme (AoE/P-10/01-1-A).

\section{REFERENCES}

1. J. Kido, J. Phys. World 12, 27 (1999).

2. J. L. Bredas and R. Silbey, Conjugated Polymers: The Novel Science and Technology of Highly Conducting and Nonlinear Optically Active Materials (Kluwer Academic Publishers, Dordrecht, 1991).

3. Y. M. Huang, J. W. Y. Lam, W. Ge, and B. Z. Tang, Appl. Phys. Lett. 75, 4094 (1999).

4. Y. Huang, W. Ge, J. W. Y. Lam, and B. Z. Tang, Appl. Phys. Lett. 78, 1652 (2001).

5. Y. Huang, W. Ge, J. W. Y. Lam, K. K. L. Cheuk, and B. Z. Tang, Mater. Sci. Eng. B 85, 122 (2001).

6. J. W. Y. Lam and B. Z. Tang, J. Polym. Sci., Part A: Polym. Chem. 41, 2607 (2003).

7. J. W. Y. Lam, Y. Dong, and B. Z. Tang, Polym. Prepr. 42(2), 193 (2001).

8. J. Luo, Z. Xie, J. W. Y. Lam, L. Cheng, H. Chen, C. Qiu, H. S. Kwok, X. Zhan, Y. Liu, D. Zhu, and B. Z. Tang, Chem. Commun. 1740 (2001).

9. M. Freemantle, Chem. Eng. News. 79(41), 29 (2001).

10. B. Z. Tang, X. Zhan, G. Yu, P. P. S. Lee, Y. Liu, and D. Zhu, J. Mater. Chem. 11, 2874 (2001).

11. H. Chen, J. W. Y. Lam, J. Luo, Y. Ho, B. Z. Tang, D. Zhu, M. Wong, and H. S. Kwok, Appl. Phys. Lett. 81, 574 (2002).

12. J. Chen, C. C. W. Law, J. W. Y. Lam, Y. Dong, S. H. F. Lo, I. D. Williams, D. Zhu, and B. Z. Tang, Chem. Mater. 15, 1535 (2003).

13. J. Chen, Z. Xie, J. W. Y. Lam, C. C. W. Law, and B. Z. Tang, Macromolecules 36, 1108 (2003).

14. J. Chen, H. Peng, C. C. W. Law, Y. Dong, J. W. Y. Lam, I. D. Williams, and B. Z. Tang, Macromolecules 36, 4319 (2003).

15. H. Peng, J. Luo, L. Cheng, J. W. Y. lam, K. Xu, Y. Dong, D. Zhang, Y. Huang, Z. Xu, and B. Z. Tang, Opt. Mater. 21, 315 (2002).

16. K. Xu, H. Peng, Q. Sun, Y. Dong, F. Salhi, J. Luo, J. Cheng, Y. Huang, D. Zhang, Z. Xu, and B. Z. Tang, Macromolecules 35, 5821 (2002).

17. H. Peng, L. Cheng, J. Luo, K. Xu, Q. Sun, Y. Dong, F. Salhi, P. P. S. Lee, J. Chen, and B. Z. Tang, Macromolecules 35, 5349 (2002). 
18. J. W. Y. Lam, J. Luo, H. Peng, Z. Xie, K. Xu, Y. Dong, L. Cheng, C. Qiu, H. S. Kwok, and B. Z. Tang, Chin. J. Polym. Sci. 19, 585 (2001).

19. M. D. Curtis, J. Am. Chem. Soc. 91, 6011 (1969).

20. Q. Sun, K. Xu, J. W. Y. Lam, J. A. P. Cha, X. Zhang, and B. Z. Tang, Mater. Sci. Eng. C. 16, 107 (2001).

21. Q. Sun, J. W. Y. Lam, K. Xu, H. Xu, J. A. P. Cha, P. C. L. Wong, G. Wen, X. Zhang, X. Jing, F. Wang, and B. Z. Tang, Chem. Mater. 12, 2617 (2000).

22. Q. Sun, K. Xu, H. Peng, R. Zheng, J. Chen, C. C. W. Law, M. Häußler, and B. Z. Tang, Macromolecules 36, 2309 (2003).

23. T. Masuda, M. Takatsuka, B. Z. Tang, and T. Higashimura, J. Membr. Sci. 49, 69 (1990).

24. B. Z. Tang, T. Masuda, and T. Higashimura, J. Polym. Sci. Polym. Phys. Ed. 27, 1261 (1989).

25. T. Masuda, Y. Iguchi, B. Z. Tang, and T. Higashimura, Polymer 29, 2041 (1988).

26. T. Masuda, B. Z. Tang, and T. Higashimura, Polym. J. 18, 565 (1986).

27. J. Malkin, Photophysical and Photochemical Properties of Aromatic Compounds (CRC Press, Boca Raton, FL, 1992).

28. K. S. Wong, H. Wang, and G. Lanzani, Chem. Phys. Lett. 288, 59 (1998). 